\title{
Petulant cellular acts: destroying the ECM rather than creating it
}

\author{
Stephen M. Krane
}

Department of Medicine, Harvard Medical School and the Medical Services (Arthritis Unit), Massachusetts General Hospital, 55 Fruit Street, Boston, Massachusetts 02114, USA.

Phone: (617) 726-2870; Fax: (617) 726-2872; E-mail: krane.stephen@mgh.harvard.edu.

What is called osteoarthritis is the most common type of joint disease in humans. This term is doubly unsatisfactory, first because it implies that a bony abnormality, consisting of subchondral dense bone and/or osteophytes, is prominent when in fact it is often absent, and second because it does not describe a single disease. Osteoarthritis is usually used to describe the clinical and pathological changes that are the end result of a number of different disorders (1) that lead to degradation of the ECM of the articular cartilages and therefore to loss of function of the articular cartilages in joint movement and weight bearing.

The capacity of the articular cartilages to withstand repeated motion and loading is ascribable to the unique ECM produced by the component chondrocytes. The uniqueness of the ECM in turn results from the interaction of the fibrillar and nonfibrillar components. The fibrillar components comprise the collagens, types II, IX, and $\mathrm{XI}$; nonfibrillar components include the major large proteoglycan aggrecan, other small proteoglycans, and a number of noncollagenous proteins. Most of these components are important for normal function of articular cartilages since spontaneous and/or engineered mutations in the corresponding genes result in disturbances that range from mild to lethal.

It was shown first in the 1960s, shortly after the discovery of animal collagenases in tadpoles (2), that collagenases are produced by cultured fragments of inflamed synovial tissue and that they occur in synovial fluid aspirated from involved joints in inflammatory joint diseases, such as rheumatoid arthritis $(3,4)$. Later, it was found that the major source of the collagenase was the fibroblast-like cells in the synovium (5, $6)$. It was recognized at the time that neutrophils also produced a collagenase (7). Over the past three decades numerous studies have shown that the production of collagenase and other enzymes by synovial cells or chondrocytes is stimulated, predominantly via transcriptional mechanisms, by inflammatory cytokines, particularly IL- $1 \alpha$, IL-1 $\beta$, and TNF- $\alpha$, but by other ligands as well (8). Furthermore, mesenchymal cells can be induced to produce collagenases by integrin-mediated interactions with components of the ECM (9). Thus, cells that at one stage function to synthesize the ECM may at another stage, influenced by ligands in the environment or the ECM itself, destroy the ECM. Production of enzymes capable of destroying the ECM is thought to be a major event in osteoarthritis as well as rheumatoid arthritis.

The collagenases that act at neutral $\mathrm{pH}$ are matrix metalloproteinases (MMPs), members of a subfamily of more than 20 proteinases, each of which is the product of a different gene. Structural features shared among the MMPs include a catalytic zinc-binding domain with a conserved sequence motif His Glu xxx xxx His $(10,11)$. Although there is considerable conservation of amino acid sequences and sequence motifs among the MMPs from humans and other species, only the products of specific MMP genes have the capacity to cleave native, undenatured, interstitial collagens (types I, II, III, and X) within the triple helical domain. Of the human MMPs, three were initially described as collagenases (MMP-1, -8, and -13, [collagenase-1, -2 , and -3 respectively]), although other MMPs (gelatinase A [GelA or $72 \mathrm{kDa}$ gelatinase] or MMP-2 and MT1-MMP or MMP-14) can also function as collagenases in vitro. The collagenolytic activity of GelA was demonstrated by using recombinant protein or, after purifying the enzyme free of the tissue inhibitor of MMPs (TIMP) (12). An expressed soluble form of MT-1MMP has also been shown to behave as a collagenase (13).

These MMPs (MMP-1, $-2,-8,-13$, and -14) all cleave triple helical interstitial collagens in vitro at the same locus. In type I collagen, the cleavage occurs between Gly775/Ile ${ }_{776}$ in the $\alpha 1$ (I) chain and a corresponding Gly/Leu in the $\alpha 2$ (I) chain, three quarters of the distance from the $\mathrm{NH}_{2}$-terminus, to yield a larger (A) fragment and a smaller (B) fragment (14). Each of these collagenases cleaves types II and III collagens at sites with similar amino acid sequences. It should be noted that the orthologues of human MMP-2, $-8,-13$, and -14 , but not MMP-1, have been identified in the mouse. MMP-1, $-2,-8$, and -13 are secreted as inactive zymogens that must be activated to function as proteinases.

In the report in this issue of the JCI, Neuhold et al. (15) introduced a constitutively active form of one of these collagenases, human MMP-13, into transgenic mice, allowing it to be expressed postnatally under control of the rat Col2a1 promoter. Their aim was to demonstrate whether high levels of collagenase active in articular cartilage would reproduce features of the pathology of human osteoarthritis. They showed that high levels of active collagenase were present in chondrocytes associated with excessive degradation of the type II collagen in the ECM. Depletion of proteoglycan was also evident based on the loss of safranin red $\mathrm{O}$ staining of the articular cartilages in the transgenic mice, similar to what is seen in different types of human osteoarthritis. The findings are not unexpected, since many studies have shown that collagenases have the described effects in vitro and that local stimulation of collagenase expression, using LPS endotoxin injections into the joints, induces inflammatory cytokines and provokes similar histological changes in cartilage and cleavage of type II collagen (16). The present findings emphasize, however, that changes characteristic of osteoarthritis can be produced by expression of active MMP-13 alone.

It should be noted that MMP-13, whether of rat, mouse, or human ori- 
gin, is a promiscuous proteinase and can cleave substrates other than type II collagen (17). MMP-13 is distinguished from MMP-1 and other collagenases by its ability to cleave type I collagen at a site in the $N$-telopeptide $(18,19)$, just downstream from the putative crosslinking lysine residue. Osteoblasts and fibroblastic cells can also potentially regulate collagen resorption by specifically removing ambient MMP-13 through binding and internalization (20). The specific binding of MMP-13 in osteoblastic and fibroblastic cells is mediated by a high affinity $170 \mathrm{kDa}$ protein receptor on the cell surface, but internalization requires the cooperative action of the LDL receptor-related protein (21). Chondrocytes might also possess this MMP-13 receptor system that is presumably involved in MMP-13 clearance or scavenging, but it remains possible that the receptor could also have a role in some form of cell signaling. In the model of Neuhold et al. (15), levels of active MMP-13, probably in considerable excess over those found spontaneously in osteoarthritis and unopposed by sufficient TIMP, might saturate the MMP-13 receptor system or alter cell function in other ways. These could include modulating production or action of the other collagenases and/or enzymes of the ADAMTS family that have potent aggrecanase activity $(22,23)$. The products of collagenase cleavage might also have important effects on cells, such as influencing the synthesis of the ECM itself. High levels of active MMP-13 might thus directly or indirectly trigger a host of events in cartilage, the end result of which are pathological changes that look like those of osteoarthritis.

1. Dieppe, P. 1998. Osteoarthritis. Acta Orthop. Scand. 281(Suppl.):2-5.

2. Gross, J., and Lapiere, C. 1962. Collagenolytic activity in amphibian tissues: a tissue culture assay. Proc. Natl. Acad. Sci. USA. 48:1014-1022.

3. Evanson, J.M., Jeffrey, J.J., and Krane, S.M. 1967. Human collagenase: identification and characterization of an enzyme from rheumatoid synovium in culture. Science. 158:499-502.

4. Harris, E.D., Jr., DiBona, D.R., and Krane, S.M. 1969. Collagenases in human synovial fluid. J. Clin. Invest. 48:2104-2113.

5. Dayer, J.-M., et al. 1976. Production of collagenase and prostaglandins by isolated adherent rheumatoid synovial fibroblasts. Proc. Natl. Acad. Sci. USA. 73:945-949.

6. Krane, S.M. 1981. Heberden Oration 1980: aspects of the cell biology of the rheumatoid synovial lesion. Ann. Rheum. Dis. 40:433-448.

7. Lazarus, G.S. et al. 1968. Human granulocyte collagenase. Science. 159:1483-1485

8. Firestein, G.S. 1996. Etiology and pathogenesis of rheumatoid arthritis. In Textbook of rheumatology. 5th edition. W.N. Kelley, E.D. Harris, Jr., S. Ruddy, and C.B. Sledge, editors. W.B. Saunders. Philadelphia, Pennsylvania, USA. 851-897.

9. Werb, Z. 1997. ECM and cell surface proteolysis: regulating cellular ecology. Cell. 91:439-442.

10. Nagase, H., and Woessner, J.F., Jr. 1999. Matrix metalloproteinases. J. Biol. Chem. 274:21491-21494.

11. Bode, W. et al. 1999. Structural properties of matrix metalloproteinases. Cell. Mol. Life Sci. 55:639-652.

12. Aimes, R.T., and Quigley, J.P. 1995. Matrix metalloproteinase- 2 is an interstitial collagenase. Inhibitor- free enzyme catalyzes the cleavage of collagen fibrils and soluble native type I collagen generating the specific 3/4- and 1/4-length fragments. J. Biol. Chem. 270:2446-2451.

13. Ohuchi, E., et al. 1997. Membrane type 1 matrix metalloproteinase digests interstitial collagens and other extracellular matrix macromolecules. J. Biol. Chem. 272:2446-2451.

14. Gross, J. 1981. An essay on biological degradation of collagen. In Cell biology of the extracellular matrix. E.D. Hay, editor. Plenum. New York, New York, USA. 217-258.

15. Neuhold, L.A., et. al. 2001. Postnatal expression in hyaline cartilage of constitutively active human collagenase-3 (MMP-13) induces osteoarthritis in mice. J. Clin. Invest. 107:35-44.

16. Otterness, I.G. et al. 1999. Detection of collagenaseinduced damage of collagen by $9 \mathrm{~A} 4$, a monoclonal C-terminal neoepitope antibody. Matrix Biol. 19:331-341.

17. Pendàs, A.M., et al. 2000. An overview of collagenase-3 expression in malignant tumors and analysis of its potential value as a target in antitumor therapies. Clin. Chim. Acta. 291:137-155.

18. Liu, X., et al. 1995. A targeted mutation at the known collagenase cleavage site in mouse type I collagen impairs tissue remodeling. J. Cell Biol. 130:227-237.

19. Krane, S.M., et al. 1996. Different collagenase gene products have different roles in degradation of type I collagen. J. Biol. Chem. 271:28509-28515.

20. Omura, T.H., et al. 1994. Identification of a specific receptor for interstitial collagenase on osteoblastic cells. J. Biol. Chem. 269:24994-24998.

21. Barmina, O.Y., et al. 1999. Collagenase-3 binds to a specific receptor and requires the low density lipoprotein receptor-related protein for internalization. J. Biol. Chem. 274:30087-30093.

22. Tortorella, M.D., et al. 1999. Purification and cloning of aggrecanase-1: a member of the ADAMTS family of proteinases. Science. 284:1664-1666

23. Tortorella, M., et al. 2000. The thrombospondin motif of aggrecanase-1 (ADAMTS-4) is critical for aggrecan substrate recognition and cleavage. J. Biol. Chem. 275:25791-25797. 